\title{
Correction to: Novel adjuvant dendritic cell therapy with transfection of heat-shock protein 70 messenger RNA for patients with hepatocellular carcinoma: a phase $\mathrm{I} / \mathrm{Il}$ prospective randomized controlled clinical trial
}

\author{
Hiroto Matsui ${ }^{1} \mathbb{D}$. Shoichi Hazama ${ }^{1,2} \cdot$ Masao Nakajima $^{1} \cdot$ Ming Xu$^{1} \cdot$ Satoshi Matsukuma ${ }^{1} \cdot$ Yukio Tokumitsu $^{1}$. \\ Yoshitaro Shindo ${ }^{1}$ - Shinobu Tomochika ${ }^{1}$. Shin Yoshida ${ }^{1}$. Michihisa lida ${ }^{1} \cdot$ Nobuaki Suzuki $^{1} \cdot$ Shigeru Takeda $^{1}$. \\ Shigefumi Yoshino ${ }^{1,3} \cdot$ Tomio Ueno $^{4} \cdot$ Masaaki Oka $^{5} \cdot$ Hiroaki Nagano $^{1}$
}

Published online: 5 January 2021

(c) Springer-Verlag GmbH Germany, part of Springer Nature 2020

\section{Correction to: Cancer Immunology, Immunotherapy https://doi.org/10.1007/s00262-020-02737-y}

Unfortunately, the given name and family name of the first author was incorrectly tagged in the $\mathrm{xml}$ data, therefore it is abbreviated wrongly in Pubmed. The correct given name is Hiroto and family name is Matsui.
Publisher's Note Springer Nature remains neutral with regard to jurisdictional claims in published maps and institutional affiliations.

The original article can be found online at https://doi.org/10.1007/ s00262-020-02737-y.

Hiroaki Nagano

hnagano@yamaguchi-u.ac.jp

1 Department of Gastroenterological, Breast

and Endocrine Surgery, Yamaguchi University Graduate

School of Medicine, 1-1-1 Minami-Kogushi, Ube,

Yamaguchi 755-8505, Japan

2 Department of Translational Research and Developmental Therapeutics Against Cancer, Yamaguchi University School of Medicine, Ube, Yamaguchi, Japan

3 Yamaguchi University Hospital Cancer Center, Ube, Yamaguchi, Japan

4 Department of Digestive Surgery, Kawasaki Medical School, Kurashiki, Okayama, Japan

5 Yamaguchi University, Yamaguchi, Yamaguchi, Japan 